Henning, Günter; Wiechmann, Volker:

\title{
Ophthalmoinnovation Thüringen : Synergiepotential für die Augenheilkunde
}

\author{
Zuerst erschienen in: Biomedizinische Technik = Biomedical Engineering. - Berlin [u.a.] : \\ de Gruyter. - 46 (2001), S1, S. 536-537. \\ Jahrestagung der Deutschen Gesellschaft für Biomedizinische \\ Technik (DGBM) im VDE ; 35 (Bochum) : 2001.09.19-21 \\ Erstveröffentlichung: 2001 \\ Datum Digitalisierung: $\quad$ 2009-08-06 \\ ISSN (online): $\quad$ 1862-278X \\ ISSN(print) 0013-5585 \\ DOI: $\quad$ 10.1515/bmte.2001.46.s1.536 \\ [Zuletzt gesehen: 2019-12-06]
}

„Im Rahmen der hochschulweiten Open-Access-Strategie für die Zweitveröffentlichung identifiziert durch die Universitätsbibliothek IImenau."

"Within the academic Open Access Strategy identified for deposition by IImenau University Library."

„Dieser Beitrag ist mit Zustimmung des Rechteinhabers aufgrund einer (DFGgeförderten) Allianz- bzw. Nationallizenz frei zugänglich."

"This publication is with permission of the rights owner freely accessible due to an Alliance licence and a national licence (funded by the DFG, German

Research Foundation) respectively."

\section{DFG}

Nationallizenzen 


\title{
OPIITHALMOINNOVATION THÜRINGEN - SYNERGIEPOTENTIAL FÜR DIE AUGENHEILKUNDE
}

\author{
G. Henning', V. Wiechmann ${ }^{2}$ \\ 'Institut für Biomedizinische Technik und Informatik, TU Ilmenau \\ ${ }^{2}$ Ophthalmolnnovation Thüringen e. V., Jena \\ ghc@informatik.tu-ilmenau.de \\ kompetenz@ophthalmoinnovation.de
}

\section{MOTIVATION UND PROBLEMSTELLUNG}

Das Auge ist das wichtigste Sinnesorgan des Menschen. Eine Verminderung des Sehvermögens bzw. dessen Verlust stellt eine erhebliche Beeinträchtigung der Lebensqualität dar und führt in der zunehmend audiovisuell geprägten Arbeitsumwelt zu einer starken Minderung der Erwerbsfähigkeit. Die gesundheitsökonomischen Auswirkungen ophthalmologischer Erkrankungen sind daher außergewöhnlich hoch.

Bei der Diagnose und Therapie von Augenerkrankungen spielen optische Methoden eine entscheidende Rolle. Technologien, die zur exakten Vermessung des optischen Systems und bei Veränderung dieses optischen Systems zur Verbesserung des Sehvermögens führen, eröffnen eine breite Anwendung mit hoher Akzeptanz bei den Patienten.

Aufgrund der Verschiebung der Alterspyramide werden Technologien zur Erhaltung des Sehvermögens bei Augenerkrankungen in der Zukunft in zunehmendem Maße benötigt. Daher wächst auch der Bedarf des
Weltmarkts an innovativen Lösungen für die ophthalmologische Diagnostik und Therapie deutlich überproportional. Aus dieser Situation resultiert die Motivation für die Entwicklung eines leistungsfähigen Netzwerks auf dem Gebiet der Medizintechnik für die Ophthalmologie.

\section{STRUKTUR DES ZENTRUMS}

Das Kompetenzzentrum OphthalmoInnovation Thüringen ist mit seiner Orientierung auf die optische Medizintechnik auf einen Schwerpunkt der Medizintechnik fokussiert, in dem dieser Verbund in der ganzen Breite des Kompetenzfeldes - von der medizintechnischen und klinischen Grundlagenforschung über die industrielle Anwendungsforschung und Entwicklung, die klinische Applikation bis zur Marktbearbeitung und gesundheitsökonomischen Evaluierung - im deutschlandweiten und auch im internationalen Vergleich eine Spitzenposition einnimmt. Ziel des Zentrums ist es, diese Position durch

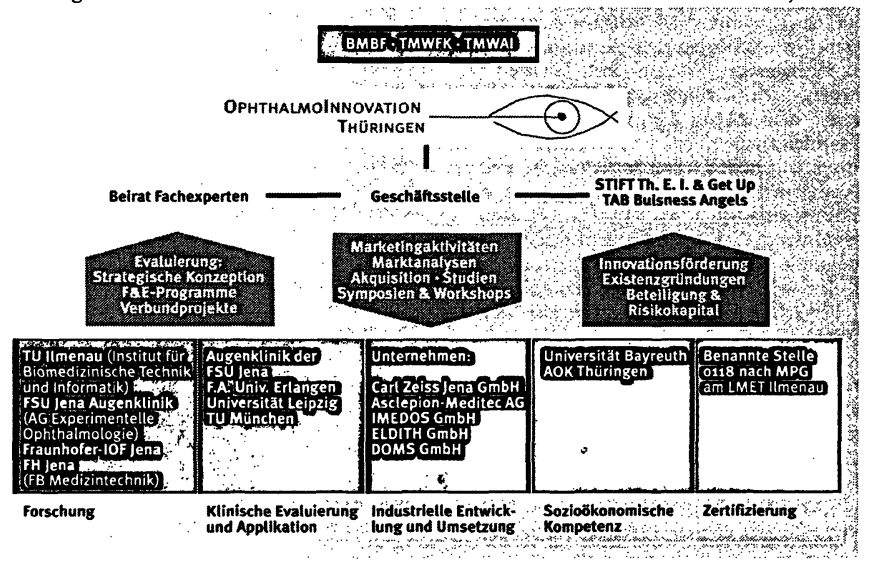

Abbildung 1: Struktur des Zentrums OphthalmoInnovation Thüringen 
Bündelung der Kräfte und Kompetenzen langfristig zu stabilisieren und auszubauen. Die Struktur des Zentrums (Abbildung 1) soll dieses Ziel effizient umsetzen. Die das Netzwerk tragenden Partner aus Wirtschaft, Klinik und Forschung haben als wettbewerbsneutralen Träger für das Kompetenzzentrum bereits Mitte 1999 einen eingetragenen Verein gegründet, dessen Geschäftsstelle neben der Gesamtkoordination aller Aktivitäten weitere Hauptaufgaben in der Akquisition von Projekten sowie der zur Realisierung der Ziele erforderlichen Mittel und ggf. weiterer Partner sowie im übergreifenden Marketing für das Zentrum übertragen wurden.

Der Geschäftsstelle steht ein Beirat mit Experten aus Medizintechnik und medizinischer Forschung sowie von Persönlichkeiten mit betriebswirtschaftlicher und gesundheitsökonomischer Kompetenz zur Seite. Kernaufgaben dieses Gremiums sind die Identifizierung und Evaluierung der im Zentrum zu bearbeitenden Projektlinien, die Begutachtung von Projektanträgen und die begleitende Evaluierung der Projektbearbeitung. Die Voten des Beirats bilden die Grundlage für Entscheidungen des Vorstands zur mittel- und langfristigen Weiterentwicklung des Zentrumskonzepts sowie (im Sinne einer empfehlenden Vorbewertung) zur Einordnung von Projektanträgen an die Fördermittelgeber.

Das Kompetenzzentrum OphthalmoInnovation Thüringen fördert die Generierung neuer technologieorientierter Untermehmensgründungen auf der Basis von Innovationen und Produktlinien, die im Verbund erarbeitet wurden. Dazu werden die Förderchancen des Projekts GetUp (Sieger im BMBFWettbewerb EXIST) sowie die hervorragende Infrastruktur, die das Applikationszentrum Ilmenau (APZ) insbesondere für Gründer im Technologiefeld Medizintechnik bietet, effektiv genutzt.

\section{INHALTLICHE SCHWERPUNKTE}

Inhaltliche Zielstellung des Zentrums ist die Neuund Weiterentwicklung innovativer Produkte und Dienstleistungen für die Ophthalmologie mit folgenden Schwerpunkten:

- Funktionsdiagnostische Systeme zur objektiven Beurteilung der Funktionen des Sehvorgangs, des Stoffwechsels und der Mikrozirkulation des Auges

- Therapeutische Systeme für die Laseranwendung in der Augenheilkunde.

Diese Schwerpunkte werden zunăchst in folgenden sieben Projektlinien umgesetzt:

- Bildgebende Basistechnologie für Funktionsimaging und individuelle Therapiefuhrung am Auge

- Funktionsimaging der Mikrozirkulation

- Spektralimaging zur Erfassung therapeutisch relevanter Kenngroßen des Stoffwechsels

- Elektrodiagnostik zur objektiven Beurteilung des visuellen Funktionszustandes

- Refraktive Laserchirurgie: Topografie- und Wellenfrontanalyse-gestützte Laserablation der Cornea mit online-Kontrolle
- Lasertherapeutische Kataraktbehandlung; LaserMikrochirurgie am Auge

- Neue Konzepte der Lasertherapie: MikrosekundenLaser, Photodynamische Therapie.

Die Auswahl dieser Schwerpunkte ist durch die möglichen Synergieeffekte in diagnostischer bzw. therapeutischer, naturwissenschaftlicher, gerätetechnischer und marktbezogener Relevanz bestimmt. Die definierten Projektlinien konzentrieren die in der Region vorhandenen Kompetenzen und Potentiale aus Forschung, Industrie und Klinik auf hochinnovative methodische und Geräteentwicklungen, die eine Stabilisierung und deutliche Erweiterung der internationalen Marktpositionen der beteiligten Unternehmen erwarten lassen.

\section{PERSPEKTIVE DES ZENTRUMS}

Schwerpunkt der Arbeit des Kompetenzzentrums in der Startphase ist der Aufbau einer effektiven Struktur der Zusammenarbeit der beteiligten Partner. Durch Einbindung weiterer Partner des bestehenden technologischen Umfeldes der Region und darüber hinaus sollen Reserven aufgedeckt und weitere Synergien erschlossen werden.

Die inhaltliche und strukturelle Orientienung des Kompetenzzentrums wird während der Bearbeitung der geplanten Aufgabenstellungen ständig an die Bedürfnisse des Marktes und an die sozioökonomischen und gesundheitspolitischen Erfordemisse bezüglich Kostensenkung und Qualitătsverbesserung angepasst.

Das Netzwerk Ophthalmolnnovation Thüringen ist auf Nachhaltigkeit orientiert: die Arbeit des Zentrums muss nach Ablauf des Förderzeitraums so effektiv sein, dass die Institution Kompetenzzentrum eine von den Partnem und Dritten anerkannte Einrichtung ist, die sich durch Beiträge der Partner und durch Einwerbung von Drittmitteln selbst finanziell trägt.

\section{DANKSAGUNG}

Für die Förderung des Kompetenzzentrums Ophthalmolnnovation Thüringen danken wir

- dem Bundesministerium für Bildung und Forschung (BMBF)

- dem Thüringer Ministerium für Wissenschaft. Forschung und Kunst (TMWFK)

- dem Thüringer Ministerium für Wirtschaft. Arteit und Infrastruktur (TMWAI)

- der Stiftung für Technologic- und Innovationsforderung Thüringen (STIFT).

Für die konstruktive und verstăndnisvolle Begleitung in der Anlaufphase gilt unser Dank dem Projekttrăger beim VDI-Technologiezentrum Dusscldorf. 